\title{
Implications for cognitive quantum computation and decoherence limits in the presence of large extra dimensions
}

\author{
J. R. Mureika \\ Department of Physics, Loyola Marymount University \\ 1 LMU Drive, Los Angeles, CA 90045-8227 \\ email: jmureika@lmu.edu
}

\begin{abstract}
An interdisciplinary physical theory of emergent consciousness has previously been proposed, stemming from quantum computation-like behavior between $10^{9}$ or more entangled molecular qubit states (microtubulin). This model relies on the Penrose-Diósi gravity-driven wavefunction collapse framework, and thus is subject to any secondary classical and quantum gravity effects. Specifically, if large extra spatial dimensions exist in the Universe, then the resulting corrections to Newtonian gravity cause this model to suffer serious difficulties. It is shown that if the extra dimensions are larger than $100 \mathrm{fm}$ in size, then this model of consciousness is unphysical. If the dimensions are on the order of $10 \mathrm{fm}$ in size, then a significantly smaller number of microtubulin than originally predicted are required to satisfy experimental constraints. Some speculation on evolution of consciousness is also offered, based on the possibility that the size of these extra dimensions may have been changing over the history of the Universe.
\end{abstract}

PACS 87.16.Ka, 03.67.Lx, 04.50.+h 


\section{Introduction}

Consciousness as an emergent phenomenon of physical and/or biological systems is a growing field of interdisciplinary interest. Traditionally, biophysical and neuroscientific descriptions of brain processes have been restricted to classical neural network designs (see e.g. [1] and references therein). However, there is increasing evidence to suggest that many biological processes rely on quantum mechanical behavior, including protein folding [2], singlephoton activation of the rhodopsin cis-trans isomers in visual pre-perception [3], and neuron impulse transmission across the synaptic gap [4.

The quantum computing revolution [5] has spawned a new frame of reference from which to view the problem of consciousness. To reconcile the aforementioned "bio-quantum mechanical" phenomena with the common interpretation of the brain as a computational device, recent proposals have sought to describe higher cognitive functions as quantum processes. Comprehensive summaries of this burgeoning field can be found in Reference [6], but specific important contributions to the field include [7, 8, 9, 10, 11, 12. From a slightly different perspective, Davies 13 has proposed a link between biological complexity and holographic theories of cosmological entropy bounds.

Classical computation is based on a binary system of bits which must either be in one state (1) or the other (0). Memory storage and computation proceed by assigning values to a string of bits and performing logical operations in series. Such a "linear" processing system ultimately limits the speed with which calculations can be performed. Quantum computers export the superposition principle of states, replacing the classical bit with a two-state quantum bit (qubit) $|\psi\rangle$, which can assume both values (0 and 1) simultaneously. Quantum logic operations are performed on the combination of qubits in the product state $\left|\psi_{1}\right\rangle \otimes\left|\psi_{2}\right\rangle \otimes\left|\psi_{3}\right\rangle \otimes \cdots$, which force evolution to an entangled superposition of states. Since this enables the quantum computer to evaluate multiple and simultaneous "solutions" to the logical operations, massively-parallel computations can be effected.

Among the many immediate benefits of quantum computing is the decreased computation time required for classically-difficult problems. This includes prime number factorization algorithms that execute in polynomial time as opposed to exponential time [14 (as a function of the size of the number), as well as fast database search algorithms [15]. Both of these are particularly attractive features to cognitive scientists, since the classical computational time required to perform equivalent tasks is astronomical. 
The primary obstacle to a realization of quantum computation is preventing the qubit entanglement from decohering via interactions with the surrounding environment. This is generally done by isolating the system from the environment, and reducing the temperature to exceedingly low values, making the notion of room-temperature (and desktop) quantum computers a distant reality.

Such rapid decoherence is arguably the death-knell for most quantum theories of cognition. The academic community is split on whether or not this is an obstacle that Nature has managed to overcome in designing a biological quantum computer (see the discussion in [16]). The most developed theory of cognitive quantum computation - dubbed the "Orchestrated Objective Reduction" (Orch-OR) mechanism - has been proposed by Penrose and Hameroff [12], who argue that environmentally-isolated quantum entanglement states are kept coherent long enough to perform conscious processes.

However, the Orch-OR mechanism relies on classical Newtonian gravity to address a largely quantum mechanical issue. If a theory wishes to combine gravitation with quantum effects, then all aspects of quantum gravity should be addressed. In particular, the accuracy of Newtonian gravity at small (submicron) scales has recently come under scrutiny, thanks to string-inspired theories which propose the existence of large extra spatial dimensions [17, 18.

This paper will thus re-examine the feasibility of the Orch-OR in light of the possible existence of large extra spatial dimensions (in the Arkani-Hamed, Dvali, Dimopoulos model). First, a more detailed synopsis of the debate surrounding the Orch-OR model are discussed in Section 2. The physical basis for the gravity-driven collapse mechanism is reviewed in Section 3, and a brief introduction to large extra dimensions follows in Section 5 . Associated modifications to the gravity-collapse model are discussed in Section [6, including the potential impact on "traditional" quantum mechanical phenomena such as nucleon superposition. Finally, the impact on Orch-OR is addressed in Section [7. It is shown that the decoherence times calculated in [12] are greatly affected by such a supposition. Should extra dimensions exist and be of sufficiently large scale, the Orch-OR scenario could suffer serious setbacks.

Before proceeding, it should be emphasized from the outset that the analysis herein is based on a combination of both hypothetical and arguably untraditional models (Orch-OR), as well as accepted but untested theory (large extra dimensions). For the sake of this argument, it is assumed that the Orch-OR model is correct. The cautious reader should thus approach the paper as an exercise in academic discourse and open-minded speculation. 


\section{A summary of the debate: Orch-OR or not Orch-OR?}

The "Orchestrated Objective Reduction" (Orch-OR) mechanism posits that conscious "computation" does not take place in the classical neural circuitry of the brain, but rather in the constituent molecules (microtubules) of the cellular cytoskeleton. Microtubules are hollow, cylindrical structures whose walls consist of 13 chains of the protein tubulin. These proteins can assume two distinct physical conformations resulting from different electric dipole moments along their physical axis. Consequently, this "two-state" behavior suggests that the tubulin protein is a prime candidate for a qubit. Penrose [12] has proposed that each tubulin qubit can become entangled with other local tubulin to form a superposition state. These superpositions are unstable and subject to collapse, and the mechanism which drives this collapse has been proposed to be that of Penrose [19, 20, 21], coined "objective reduction".

The primary criticism of this model is that the brain is not an isolated low temperature environment, and thus the decoherence of any macroscopic entanglements would be effectively instantaneous $\left(\sim 10^{-20}\right.$ sec $)$ due to other local quantum processes such as ion interactions [16. This criticism is countered by arguing that the microtubules are sufficient shielded by molecular and electrostatic considerations, thus the entangled states can survive for macroscopic time intervals 22 .

Furthermore, the authors of [12] suggest that well-known electrophysiological "brain-wave" frequencies are signatures of the extended superposition collapse. For example, they demonstrate that the Orch-OR mechanism allows tubulin qubit superpositions to be maintained for as long as 25 microseconds, corresponding to the $40 \mathrm{~Hz}$ thalamo-cortical coherent oscillation frequency. This association (among others) has been offered as evidence in support of the quantum computing model for consciousness.

In the Orch-OR model, the culprit for the wavefunction collapse is gravity. Penrose [19, 20] has postulated that gravitational interactions between the different physical configuration of each qubit eigenstate introduces a type of time-energy uncertainty relationship. This ultimately limits the duration that a state can remain in superposition, and is discussed in detail in the next section. 


\section{The physical foundations of Objective Re- duction}

The Objective Reduction (OR) mechanism is a potential solution to the measurement problem between the two (or more) possible eigenstates of an evolving wavefunction, $|\Psi\rangle=\alpha\left|\psi_{1}\right\rangle+\beta\left|\psi_{2}\right\rangle$. A complete derivation of the OR mechanism will not be reproduced here, but can be found in References 19, 20, 21]. Similar collapse schemes have been proposed by other authors [23. 24, 25, 26, but will not be discussed in the present context ${ }^{1}$. A review of four distinct interpretations of such collapse mechanisms may be found in 27.

The theory proposes that each eigenstate in the superposition possesses a conformationally-distinct physical orientation. The overlap of both conformations will have a small but relevant impact on the local curvature of spacetime. The net result is that there will be two distinct spacetime curvatures in superposition with one another. Each curvature can be represented by a quantum state $\left|\mathcal{G}_{i}\right\rangle$, correlated with the eigenstate $\left|\psi_{i}\right\rangle$, and thus the actual particle wavefunction will be represented by the entanglement $\left|\psi_{1}\right\rangle\left|\mathcal{G}_{1}\right\rangle+\beta\left|\psi_{2}\right\rangle\left|\mathcal{G}_{2}\right\rangle$. The geometric superposition creates an ill-definition of the time-like Killing vector, $\partial / \partial t$, which will ultimately lead to the collapse. Simply put, each eigenstate will follow its own unique free-fall vector, simultaneously violating the weak equivalence principle.

It can be shown that this "instability" in the superposition is limited by an upper bound on the gravitational interaction energy between the two eigenstate conformation states. Assuming the separation of each state $(\Delta r)$ exceeds their own physical extent, this is simply the Newtonian energy

$$
E_{\Delta} \sim \frac{G m^{2}}{\Delta r}
$$

The states can just as easily overlap physically, although the calculation becomes more complicated. The collapse time of the spacetime superposition is determined by the uncertainty relation

$$
T_{c} \sim \frac{\hbar}{E_{\Delta}}
$$

\footnotetext{
${ }^{1}$ It should be pointed out that the mechanism proposed in 23 . predates and ultimately produces similar results to Penrose's formalism.
} 
and thus is inversely proportional to the gravitation energy of the system. This result is a consequence of the framework in [23, as well as the alternate derivation in [19, 20, 21, and the interested reader is directed to these References for a complete review of the foundations.

Hence, in this scheme a nucleon of mass $10^{-27} \mathrm{~kg}$ whose superpositions are separated by the strong interaction scale of $10^{-15} \mathrm{~m}$ will remain superposed for $T_{c} \sim 10^{15}$ seconds (or about $10^{7}$ years), whereas a possible macroscopic superposition having larger $E_{\Delta}$ will decay relatively quickly. For example, if a speck of dust $\left(m \sim 10^{-6} \mathrm{~kg}\right.$ ) evolves into superposed states that are separated by $0.01 \mathrm{~mm}$, the wavefunction would collapse in well under $10^{-16}$ seconds.

\section{Orchestrated Objective Reduction}

Orchestrated Objective Reduction (Orch-OR) is the application of the OR formalism to superpositions of entangled tubulin "qubits" in the cellular cytoskeleton. Using this rationale, the authors of [12] have calculated a variety of constraints on the nature of tubulin structures in which conscious quantum computations could be realized. Various electrophysiological frequencies are known to exist in the brain - most notably the $40 \mathrm{~Hz}$ thalamo-cortical coherent oscillations $(\Delta t=25 \mathrm{~ms}), 10 \mathrm{~Hz}$ alpha rhythm EEG $(\Delta t=100 \mathrm{~ms})$, and Libet's pre-conscious $2 \mathrm{~Hz}$ sensory threshold $(\Delta t=500 \mathrm{~ms})$ [12]. If this period $\Delta t$ corresponds to the collapse time for tubulin qubit entanglements, then it can be reasoned that $\Delta E \sim \frac{\hbar}{\Delta t} \approx 10^{-15} \mathrm{eV}$ is the required gravitational self-energy which must be attained by the system for the longest period of $500 \mathrm{~ms}$. This implies that $N$ qubits possessing an OR energy $E_{0}$ must be entangled together, where $\Delta E=N E_{0}$.

In order to estimate the self-energy $E_{0}$ of one tubulin superposition, it has been suggested [12] that the most appropriate conformation states are those in which a carbon-12 nucleus is superposed within its own atomic radius ( $a \sim 2.5 \times 10^{-15}$ meters). In this case, the self-energy term becomes

$E_{0}=\frac{G m_{C}^{2}}{a} \sim 10^{-28} \mathrm{eV}$, and thus $N=\Delta E / E_{0} \sim 10^{13}$ carbon atoms, or $10^{14}$ nucleons. Since each microtubule is composed of $\mu \sim 10^{5}$ nucleons, this implies that $N_{T} \sim 10^{9}$ tubulin are required for a conscious instance. This corresponds to roughly 100 neurons, each containing $10^{7}$ tubulin [28].

Combining these into one single expression, it can be concluded that the number of microtubulin $N_{T}$ required to form a $\Delta t$ second pre-conscious 
instance is

$$
N_{T} \sim \frac{\hbar a}{\mu G m_{C}^{2} \Delta t}
$$

\section{$5 \quad$ Large Extra Spatial Dimensions}

It is a long-standing supposition in theoretical physics that our Universe might contain more that the traditional three spatial dimensions. The initial proposal made by Kaluza [29] and later by Klein [30] posited that a $(4+1)$ dimensional metric could help unify the fundamental fields of gravitation and electromagnetism. This extra dimension has traditionally assumed to be compactified with a small radius $R \ll 1$, which has until recently been taken to be of the Planck length $\left(>10^{-35} \mathrm{~m}\right)$.

The emergence of theories of large extra dimensions (LEDs) in the late 1990s relaxed the constraint that the size of any additional spatial dimensions must be Planck order (see [17, 18, for their pioneering works). Although introduced initially as a solution to the hierarchy problem, LED theories have found numerous applications in the fields of particle physics and cosmology, including candidates for missing energy in GeV-scale accelerator collisions 31, cosmic ray flux spectra anomalies [32, and dark energy phenomenology 33 . This manuscript will deal only with compactified Kaluza-Klein-like ADD extra dimensions [17, and not the Randall-Sundrum "warped" dimensions [18].

The energy scale (or temperature) at which gravity is expected to unify with the other fundamental forces is exceedingly large, $M_{P l} \sim 16 \mathrm{TeV}$. Such energies would have been present a brief fraction of a second after the Big Bang (as a comparison, the present background energy of the Universe is in the range of $10^{-4} \mathrm{eV}$ ). The large energy scale in turn explains the smallness of the gravitational constant, since $G_{N}=M_{P l}^{-2}$. The underlying assumption of LED theory is that the electroweak unification scale $M_{E W} \approx 1 \mathrm{TeV}$ is also the fundamental scale of gravitation $M_{4+n}$ in the full $4+n$ dimensional spacetime. Just as the electroweak coupling goes as $m_{E W}^{-2}$, the "actual" gravitational coupling $\left(G_{4+n}\right)$ is set by this new energy scale.

In $3+n$ spatial dimensions, the Newtonian potential differs from the familiar $1 / r$ form. If there are $n$ extra compactified dimensions of scale size $R_{n}$, then for distances $r<R_{n}$ it can be shown (e.g. via Gauss' Law) that

$$
\left|\phi_{n}(r)\right| \sim \frac{G_{4+n} m}{r^{n+1}},
$$


(up to some geometric constants) since the fields can now propagate in any of the $3+n$ spatial dimensions. Gravitational interactions in the full $4+n$ dimensional spacetime are mediated by the new coupling constant $G_{4+n}$, whose value is set by the $\mathrm{TeV}$ unification scale. For small $r$, the strength of gravitation becomes commensurate with the other fundamental forces, particularly electromagnetism.

Gravity's apparent weakness at macroscopic distances arises from the fact that the range of influence of the extra dimensions is limited to some distance $R_{n}$, which is just the dimensions' compactification scale. As a result, the value of the traditional Newtonian constant $G_{N}$ is fixed by the size and number of the extra dimensions.

Continuity of the gravitational field at $r=R_{n}$ requires

$$
\frac{G_{4+n} m}{R_{n}^{n+1}} \sim \frac{G_{N} m}{R_{n}}
$$

So a relationship between the two coupling constants may be derived as

$$
G_{4+n} \sim R_{n}^{n} G_{N}
$$

which indicates that the value of the compactification radius is

$$
R_{n} \sim 10^{\frac{32}{n}-19} \text { meters }
$$

As counter-intuitive as the result may seem, the traditional laws of Newtonian gravity might break down below this length scale. It can quickly be shown that if large extra compactified dimensions exist, there must be more than one. If $n=1$, then $R \sim 10^{11} \mathrm{~m}$, which would imply that deviations from Newtonian gravity should be observed at scales of the order of the solar system (which clearly they do not). Probing the cases $n \geq 2$ has subsequently become a hot topic of research, ranging from the aforementioned astrophysical observation and accelerator searches to high-precision tabletop laboratory experiments. Via Cavendish-like gravitation experiments, the Eötwash group 34 has determined that gravity behaves in the classical Newtonian manner at scales as small as $200 \mu \mathrm{m}$, but a recent result has set this limit further down to $100 \mathrm{~nm}$ [35].

\section{Penrose-Diósi OR with LEDs}

Before addressing the effect of possible LEDs on something so contrived as the Orch-OR mechanism, their influence on basic quantum mechanics is first 
addressed. In a recent paper [36], it was demonstrated that the existence of LEDs can have measurable impact on nucleon collapse times described by Penrose's orchestrated reduction paradigm ${ }^{2}$

Assuming a nucleon wavefunction evolves such that the physical conformations of two eigenstates are separated by a distance on par with the radius of the nucleon itself $\left(10^{-15} \mathrm{~m}\right)$, collapse times in the presence of LEDs become much shorter than the $10^{15}$ seconds predicted in Reference [19]. In fact, if there are between $n=2$ and 3 dimensions of compactification scale $R_{2} \sim 10^{-3} \mathrm{~m}$ to $R_{3} \sim 10^{-9} \mathrm{~m}$, the nucleon wavefunction will collapse in under $10^{-5}$ seconds. This short superposition time would destroy the quantum mechanical nature of the nucleons, and thus would have observable consequences for neutron diffraction. Thus, these cases are ruled out by experiment.

If there are 4 or 5 dimensions of scale $10^{-11} \mathrm{~m}$ and $10^{-13} \mathrm{~m}$ respectively, the collapse time increases to between $0.01-10$ seconds. These case again could easily be verified experimentally, and the result could serve to constrain the LED mechanism (if Penrose's initial collapse scheme is correct). The cases $n=6$ to $n=8$ are of interest, because the nucleon collapse times increase from $10^{7}$ seconds to $10^{15}$ seconds. It would be difficult to test whether or not a nucleon may be superposed for more than a year without succumbing to collapse, and thus it leaves the question open as to whether or not these LED parameters are physically viable.

\section{Orch-OR with LEDs}

Since particles separated by distances less than the compactification radius of any LEDs will experience "stronger" gravity, there could be significant consequences for the Orch-OR mechanism. Modifying the Penrose-Hameroff derivation from Section 4, the reduction is now calculated using a potential function of the form in Equation 4 .

Equation 3 can be modified to include LEDs by the replacement $G \longrightarrow$ $G_{4+n}$, and $a \longrightarrow a^{n+1}$, giving

$$
N_{T} \sim \frac{\hbar a^{n+1}}{\mu G_{4+n} m_{c}^{2}}
$$

\footnotetext{
${ }^{2}$ The idea that LEDs could influence gravitation collapse schemes was anecdotally mentioned in Reference [26].
} 
Table 10] shows calculated values of $N_{T}$ for $n=2$ through $n=7$ extra dimensions. The three neural frequencies mentioned in Section 4 are considered, which correspond to collapse times of $25 \mathrm{~ms}, 100 \mathrm{~ms}$, and $500 \mathrm{~ms}$. Additionally, a fourth frequency is also included which represents a $5 \mathrm{~ms}$ neural signal specific to human beings (see [12]) requiring $10^{11}$ tubulin with regular Newtonian gravity. The case $n=1$ is excluded because of the aforementioned astrophysical constraints, and $n \geq 8$ are also excluded because the size $R_{n}$ drops below the mass separation (and thus regular Newtonian gravity would resume at about this point). Experimental tests of Newton's inverse square law [34] have effectively ruled out extra dimensions above a few hundred microns $\left(10^{-4} \mathrm{~m}\right)$ in size, so it is also unlikely that $n=2$ is valid.

For the cases where $3 \leq n \leq 5$, a very startling result is observed. Since gravity is so much stronger than normal at distances $r \ll R_{n}$, the self-energy of a single nucleon superposition is larger than the total required energy $\Delta E$. For instance, if there are $n=3$ extra dimensions whose scale size is roughly $1 \mathrm{~nm}$, the ratio of the superposition self-energy of a single nucleon to the total energy required for a $500 \mathrm{~ms}$ collapse would be $E_{3} / \Delta E \sim 10^{6}$. The corresponding number of tubulin required for the $100 \mathrm{~ms}$ and $25 \mathrm{~ms}$ scenarios are also unphysical for these cases. Thus, if there are indeed 5 or less extra dimensions of the variety described by the ADD theory, then it is virtually impossible for this mechanism to be responsible for conscious correlates (as described by Penrose and Hameroff). However, as discussed in the previous section, these cases would also be ruled out by experiment.

If there are seven or more extra dimensions, then their length scale size drops below the separation of the carbon nuclei in the protein qubits, and one would expect "regular" gravity to take over (as suggested by the reported data). However, it is just below this value of $n$ that the implications of OrchOR become striking. In a universe with $n=6$ extra dimensions of scale $R_{6} \sim 10^{-14}$ meters, the number of tubulin qubits $N_{T}$ becomes physicallyrealizable. This number, however, is exceedingly small, on the order of a few hundred for the $500 \mathrm{~ms}$ case. This number increases by order of magnitude with decreasing $\Delta t$, giving $N_{T} \sim 10^{3}$ tubulin for $100 \mathrm{~ms}$ and $N_{T} \sim 10^{4}$ for $25 \mathrm{~ms}$. In this case, it would imply that effectively all biological organisms containing tubulin cytoskeletons are conscious, since as previously mentioned typical neuron contains $10^{7}$ tubulin! Thus, even microscopic organisms with a relatively small number of neurons would be conscious. While philosophers might be extremely intrigued by this conclusion which opens the doors for a re-evaluation of a being's self-awareness, the likelihood of this being a reality 
is, to say the least, suspect.

\section{Variation of $\mathrm{TeV}$ scale}

The values in Table 10 assume that the unification scale is $M_{4+n} \sim 1 \mathrm{TeV}$. However, there is nothing to suggest that it cannot be slightly larger than

this. Table 10 demonstrates how the values might change if $M_{4+n}$ shifted by a few orders of magnitude and instead is $M_{4+n} \sim 10^{\delta} \mathrm{TeV}$. The main effect of raising the unification scale is to make the compactification scale of dimensions smaller.

Table [10 shows how the number of tubulin required for the constant instances of Table [10 might change if $\delta=2$ (i.e. a unification scale of $100 \mathrm{TeV})$. Note that now only the Orch-OR mechanism will be affected for only up to $n=4$ extra dimensions before the compactification scale drops below the carbon nuclei superposition separation $a$. In fact, if there are 2 or 3 extra dimensions ( 1 is still ruled out by macroscopic gravitational phenomena) the OR framework again cannot be the driving mechanism of state collapse.

\section{Evolution of consciousness and time depen- dent LEDs}

In Reference 12] a discussion of evolution and the emergence of consciousness was raised, based on the estimation of $10^{9}$ tubulin required for pre-conscious processing. There is increasing observational evidence that the value of Newton's constant $G$ has changed over the evolution of the Universe to present day (see References [37, 38] for a pre-LED and post-LED discussion of timedependent compactification radii). From Equation 6, it can be deduced that a time-dependent constant $G(t)$ varies as $R(t)^{-n}$. So, depending on whether $G(t)$ is getting bigger or smaller with time can be related to the changing scale size of the extra dimensions.

In fact, it can easily be shown that if $G(t)$ is getting bigger, then $R(t)$ must be getting smaller. If the Orch-OR mechanism is correct, then the implications for conscious emergence are striking. As has been shown in this analysis, large values of $R$ imply either unphysical interpretations of OrchOR, or alternatively that conscious processes require only a few microtubulin 
strands. This could suggest that pre-evolutionary microbes possessed conscious though (depending on the initial size of $R(t)$, that is). Conversely, a shrinking value of $G(t)$ implies that the scale $R(t)$ is getting larger over time, implying that over large time scales more organic entities will eventually achieve consciousness.

Of course, the time scales required for a significant change in the value of $G(t)$ are on the order of a fraction of the age of the Universe, which most likely would surpass the "biological" time of species on the Earth. Indeed, it has been shown that the compactification radii have grown by less than a factor of ten in size over the history of the solar system [36]. Also, the variation of $G(t)$ is also independent of the possible existence of extra dimensions. Hence, the associated impact on such quantum mechanical brain processes would still be relevant, and thus opens intriguing speculation on how intelligence might have evolved elsewhere in the early Universe.

\section{Conclusions}

This article has examined the compatibility of Penrose and Hameroff's orchestrated objective reduction model for consciousness in light of the possible existence of large extra compactified spatial dimensions of the ADD variety. Since the basis of the objective reduction model is explicitly gravitational and Newtonian, the presence of LEDs and TeV gravity will significantly alter the conclusions drawn in Reference [12]. In fact, for extra dimensions of scale size less that $\sim 10^{-14} \mathrm{~m}$ the Orch-OR model becomes an incomplete theory. The required number of tubulin to maintain the observed conscious frequencies are either outrageously small, or even unphysical $\left(N_{T}<1\right)$.

The greatest test of TeV-gravity and LEDs will begin in 2007 when the Large Hadron Collider is brought on-line. The accelerator will problem energy scales well above the TeV boundary, and as such will provide an exciting glimpse at a range of possible new physics which exists in and beyond this energy range. If extra dimensions exist and are large compared to the Planck scale, their existence is expected to be confirmed. If they do exist, then the Orch-OR model is incomplete or incorrect. If they do not, then the mystery of consciousness and its connection to quantum gravity is possibly one step closer to being explained. 


\section{References}

[1] Arbib, M. A. (ed.), The Handbook of Brain Theory and Neural Networks, MIT press, Cambridge.(1995); Arbib, M. A. and Grethe, J. S. (eds.), Computing the Brain: A Guide to Neuroinformatics, Academic Press, San Diego (2001); Koch, C. Biophysics Of Computation: Information Processing In Single Neurons, Oxford University Press , Oxford (2004)

[2] Davydov, A. S., Biology and Quantum Mechanics, Pergamon Press, Oxford (1982)

[3] Hahn, S. and Stock, G., J. Phys. Chem. B 104 (6), 1146-1149 (2000); Kim, J. E., Tauber, M. J., and Mathies, R. A, Biophys. J. 84, 2492-2501 (2003)

[4] Beck F. and Eccles, J. C., Proc. Natl. Acad. Sci. USA 89, 311-357 (1992)

[5] Nielsen, M. A. and Chuang, I. L., Quantum Computation and Quantum Information, Cambridge University Press (2000,2004)

[6] Tuszynski, J. (ed), The Emerging Physics of Consciousness, SpringerVerlag, In press (2005)

[7] Lockwood, M., Mind, Brain and Quantum, Oxford: Oxford University Press (1989)

[8] Stapp, H. P., Mind, Matter, and Quantum Mechanics, First Edition, Springer-Verlag (1993)

[9] Page, D. N., "Mindless Sensationalism: A Quantum Framework for Consciousness", in Consciousness: New Philosophical Essays, edited by Quentin Smith and Alexandar Jokic (Oxford: Oxford University Press, 2002)

[10] Eccles, J.C., How the Self Controls its Brain, Springer-Verlag, (1994)

[11] Hodgson, D., "Quantum Physics, Consciousness, and Free Will", in The Oxford Handbook of Freewill, R. Kane (ed), (Oxford University Press) (2002) 
[12] Hameroff, S. and Penrose, R., J. Conscious. Stud. 3, 36-63 (1996); Hameroff, S. and Penrose, R. "Orchestrated Reduction Of Quantum Coherence In Brain Microtubules: A Model For Consciousness?", in Toward a Science of Consciousness - The First Tucson Discussions and Debates, Hameroff, S.R., Kaszniak, A.W. and ious. A.C. (eds), Cambridge, MA: MIT Press pp. 507-540 (1996); Hameroff, S., Phil. Trans. Roy. Soc. Lon. A 356 1869-1896 (1998); Hameroff, S. and Penrose, R., DARPA/RAND Corporation preprint (October 22, 1998)

[13] Davies, P., "Emergent biological principles and the computational resources of the universe", Complexity 10 (2), 1 (2004)

[14] Shor, P., "Polynomial-Time Algorithms for Prime Factorization and Discrete Logarithms on a Quantum Computer", in Proceedings of the 35th Annual Symposium on Foundations of Computer Science, Santa Fe, NM, Nov. 20-22 (1994); quant-ph/9508027

[15] Grover, L. K., "A fast quantum mechanical algorithm for database search", in Proceedings, 28th Annual ACM Symposium on the Theory of Computing (STOC), pages 212-219 (May 1996); quant-ph/9605043

[16] Tegmark, M., Phys. Rev. E61, 4194-4206 (2000)

[17] Antoniadis, I., Arkani-Hamed, N., Dimopolous, S. and Dvali, G. Phys. Lett. B436, 257-263 (1998); Arkani-Hamed, N., Dimopolous, S. and Dvali, G., Phys. Rev. D59 806004 (1999)

[18] Randall, L. and Sundrum, R. Phys. Rev. Lett. 83, 3370 (1999); Phys. Rev. Lett. 83, 4690 (1999)

[19] Penrose, R., Gen. Rel. Grav. 28, 581 (1996)

[20] Penrose, R., Shadows of the Mind, Oxford University Press, New York (1996)

[21] Penrose, R., Phil. Trans. R. Soc. Lond. A 356 (1998) 1927-1939

[22] Hagan, S., Hameroff, S. R., and Tuszyńsky, Phys. Rev. E 65, 061901 (2002)

[23] Diósi, L., Phys. Rev. A40, 1165-1174 (1989) 
[24] Ghirardi, G. C., Grassi, R. and Rimini, A., Phys. Rev. A42, 1057-1064 (1990)

[25] Pearle, P. and Squires, E. quant-ph/9503019

[26] Geszti, T., Phys. Rev. A69, 032110 (2004)

[27] Diósi, L., Braz. J. Phys. 35, 260-265 (2005)

[28] Yu, W. and Baas, P. W., J. Neuroscience 14 (5), 2818-2829 (1994)

[29] Kaluza, T. Sitzungsber. Preuss. Akad. Wiss. Berlin (Math. Phys.) K1, 966-972 (1921)

[30] Klein, O., Z. Phys. 37, 895-906 (1926)

[31] L3 Collaboration, Phys. Lett. B587, 16-32 (2004); CDF Collaboration, Phys. Rev. Lett. 89, 281801 (2002); CDF Collaboration, Phys. Rev. D71, 031104 (2005); DELPHI Collaboration, J. Abdallah et al., Eur. Phys. J. C38 395-411 (2005)

[32] Emparan, R., Masip, M., and Rattazzi, R., Phys. Rev. D65, 064023 (2002); Kazanas, D. and Nicolaidis, A., Gen. Rel. Grav. 35, 1117-1123 (2003)

[33] Lue, A. and Starkman, G., Phys. Rev. D67, 064002 (2003); Lue, A. Scoccimarro, R., and Starkman, G. Phys. Rev. D69, 044005 (2004)

[34] Adelberger, E. G., Heckel, B. R., and Nelson, A. E., Annu. Rev. Nucl. Part. Phys. 53, 77-121 (2003)

[35] Decca, R. S. et al., Phys. Rev. Lett. 94, 240401 (2005)

[36] Mureika, J. R., to appear in Phys. Rev. D (2006) also Mureika, J. R., "Gravitationally-Induced Quantum State Collapse with Large Extra Dimensions", in Proceedings of the 11th Canadian Conference on General Relativity and Relativistic Astrophysics, Vancouver, BC, Canada (May 19-21, 2005), on-line proceedings

[37] Marciano, W. J., Phys. Rev. Lett. 52, 489-491 (1984)

[38] Loren-Aguilar, P. et al., Class. Quant. Grav. 20, 3885-3896 (2003) 


\begin{tabular}{cc|cccc}
\hline & & \multicolumn{4}{|c}{$N_{T}$} \\
$n$ & $R_{n}$ (meters) & $T=5 \mathrm{~ms}$ & $25 \mathrm{~ms}$ & $100 \mathrm{~ms}$ & $500 \mathrm{~ms}$ \\
\hline 2 & $10^{-3}$ & $10^{-13}$ & $10^{-14}$ & $10^{-14}$ & $10^{-20}$ \\
3 & $10^{-9}$ & $10^{-9}$ & $10^{-9}$ & $10^{-10}$ & $10^{-14}$ \\
4 & $10^{-11}$ & $10^{-4}$ & $10^{-5}$ & $10^{-6}$ & $10^{-6}$ \\
5 & $10^{-13}$ & 2 & 0.5 & $10^{-1}$ & $10^{-2}$ \\
6 & $10^{-14}$ & $5 \times 10^{4}$ & $10^{4}$ & $10^{3}$ & 500 \\
7 & $10^{-15}$ & $10^{9}$ & $10^{8}$ & $5 \times 10^{7}$ & $10^{7}$ \\
\hline
\end{tabular}

Table 1: The number of tubulin proteins required for an orchestrated reduction of $\Delta t=5,25,100$, and $500 \mathrm{~ms}$ duration in Universes with $n$ extra dimensions. The case $n=1$ is ruled out by the observed behavior of macroscopic gravity, while the cases $n>7$ would reproduce the "standard" Orch-OR results.

\begin{tabular}{cc|cccc}
\hline & & \multicolumn{4}{|c}{$N_{T}$} \\
$n$ & $R_{n}$ (meters) & $T=5 \mathrm{~ms}$ & $25 \mathrm{~ms}$ & $100 \mathrm{~ms}$ & $500 \mathrm{~ms}$ \\
\hline 2 & $10^{-7}$ & $10^{-5}$ & $10^{-6}$ & $6 \times 10^{-7}$ & $10^{-7}$ \\
3 & $10^{-12}$ & 30 & 6 & 1 & 0.3 \\
4 & $10^{-14}$ & $10^{8}$ & $10^{7}$ & $10^{6}$ & $7 \times 10^{5}$ \\
\hline
\end{tabular}

Table 2: The number of tubulin proteins required for an orchestrated reduction of $\Delta t=5,25,100$, and $500 \mathrm{~ms}$ duration in Universes with $n$ extra dimensions in which the gravitational unification scale is $100 \mathrm{TeV}$. The size of each dimension $R_{n}$ is smaller than those in Table 1, and thus regular Newtonian gravity is recovered at the tubulin length scales for smaller $n$. 